\title{
Tritylamine (triphenylmethylamine) in organic synthesis; III. The synthesis of 1-aminoalkylphosphonic acids in the reaction of $N$-(triphenylmethyl)alkanimines with phosphorus trichloride in acetic acid or with phosphonic (phosphorous) acid in acetic anhydride
}

\author{
Waldemar Goldeman and Mirosław Soroka* \\ Politechnika Wrocławska, Department of Chemistry, Wybrzeże Wyspiańskiego 27, PL-50370 \\ Wroctaw, Poland \\ E-mail: miroslaw.soroka@pwr.wroc.pl
}

\begin{abstract}
The reaction of phosphorus trichloride in acetic acid or phosphonic (phosphorous) acid in acetic anhydride, with $N$-(triphenylmethyl)alkanimines gives 1-acetylaminoalkylphosphonic acids 1a-j, which after hydrolysis give 1-aminoalkylphosphonic acids $\mathbf{2 a - j}$ in good yields.
\end{abstract}

Keywords: Ammonia equivalent, aminophosphonic acids, aminoalkylation, amidoalkylation, alkanimines, Schiff bases, acyliminium ions

\section{Introduction}

In 1988 we published a paper $^{1}$ concerning the application of tritylamine (triphenylmethylamine) as an ammonia equivalent in the synthesis of $N$-(triphenylmethyl)alkanimines and 1(triphenylmethylamino)alkylphosphonates. Our method is especially useful when orthogonally protected derivatives of 1-aminoalkylphosphonic acids are needed. Moreover, the trityl group can be easily removed or replaced, and then reused again for the synthesis, what makes the tritylamine an excellent ammonia equivalent. ${ }^{2}$

Unfortunately, this method of synthesis of 1-aminoalkylphosphonates has some limitations. The first is that the $N$-(triphenylmethyl)arylmethanimines derived from aromatic aldehydes do not react with dialkyl or diaryl phosphonates. ${ }^{1 a}$ For example, $N$-(triphenylmethyl) phenylmethanimine does not react with dimethyl phosphonate, even when heated for 2 hrs at 100 ${ }^{\circ} \mathrm{C}$. This method is also rather inconvenient when one wishes to prepare just free 1aminoalkylphosphonic acid. In this case using the dialkyl phosphonate (prepared in a separate 
reaction from phosphorus trichloride and alkanol) for the synthesis, and then removing the alkyl groups by hydrolysis in the next step, makes no sense.

\section{Results and Discussion}

To avoid these unnecessary steps, we decided to use directly phosphorus trichloride ${ }^{3}$ or phosphonic acid (phosphorous acid) ${ }^{4}$ (two basic industrial phosphorus compounds), respectively in acetic acid or in acetic anhydride, for the reaction with $N$-(triphenylmethyl)alkanimines (Scheme 1).
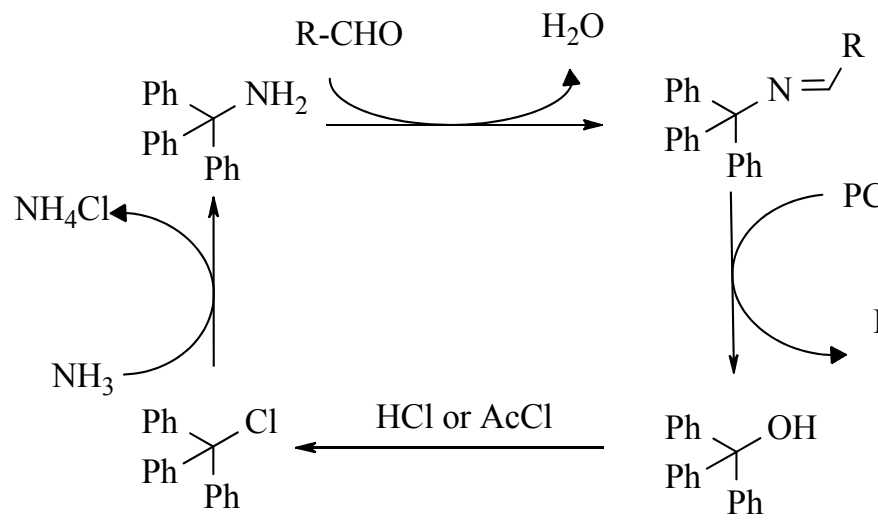

$\mathrm{PCl}_{3} / \mathrm{AcOH}$ or $\mathrm{HP}(\mathrm{O})(\mathrm{OH})_{2} / \mathrm{Ac}_{2} \mathrm{O}$<smiles>[R]C(NC(C)=O)P(=O)(O)O</smiles>

1a-j

$\mathbf{2 a - j}, 54-87 \%$

\section{Scheme 1}

We found that in most cases these reactions gave corresponding 1-aminoalkylphosphonic acids with good yields (Table). Furthermore, also $N$-(triphenylmethyl)arylmethanimines (derived from aromatic aldehydes) gave excellent yields of the desired amino(aryl)methylphosphonic acids. The only side products we found in these reactions were iminobis(alkyl-1-yl-phosphonic) acids - $\mathrm{NH}\left[\mathrm{CH}(\mathrm{R})-\mathrm{P}(\mathrm{O})(\mathrm{OH})_{2}\right]_{2}$.

Table. Yield ${ }^{\mathrm{a}}[\%]$ of isolated crystalline 1-aminoalkylphosphonic acids $\mathbf{2 a - j}$

\begin{tabular}{ccccc}
\hline No & $\mathrm{R}$ & Method $\mathrm{A}\left[\mathrm{PCl}_{3} / \mathrm{AcOH}\right]$ & Method B $\left[\mathrm{HP}(\mathrm{O})(\mathrm{OH})_{2} / \mathrm{Ac}_{2} \mathrm{O}\right]$ & Ref. \\
\hline $\mathbf{2 a}$ & $\mathrm{H}$ & 54 & 74 & $1 \mathrm{a}, 5 \mathrm{a}, 5 \mathrm{~b}$ \\
$\mathbf{2 b}$ & $\mathrm{Me}$ & 64 & 81 & $1 \mathrm{a}, 5 \mathrm{c}, 5 \mathrm{~b}$ \\
$\mathbf{2 c}$ & $\mathrm{Et}$ & 66 & 61 & $1 \mathrm{a}, 5 \mathrm{c}, 5 \mathrm{~d}$ \\
$\mathbf{2 d}$ & $\mathrm{Pr}$ & 87 & 71 & $5 \mathrm{c}, 5 \mathrm{~d}$ \\
$\mathbf{2 e}$ & $\mathrm{i}-\mathrm{Pr}$ & 66 & 71 & $1 \mathrm{a}, 5 \mathrm{~d}$ \\
$\mathbf{2 f}$ & $\mathrm{Bu}$ & 81 & 67 & $5 \mathrm{c}, 5 \mathrm{~d}$ \\
$\mathbf{2 g}$ & $\mathrm{Et}_{2} \mathrm{CH}$ & 60 & 59 & $5 \mathrm{e}$ \\
$\mathbf{2 h}$ & $\mathrm{Ph}$ & 65 & 66 & $5 \mathrm{f}, 5 \mathrm{c}, 5 \mathrm{~d}$ \\
\hline
\end{tabular}


Table. Continued

\begin{tabular}{ccccc}
\hline No & $\mathrm{R}$ & Method A [PCl3/AcOH $]$ & Method B $[\mathrm{HP}(\mathrm{O})(\mathrm{OH}) 2 / \mathrm{Ac} 2 \mathrm{O}]$ & Ref. \\
\hline $\mathbf{2 i}$ & $4-\mathrm{MeOC}_{4} 4$ & 76 & 84 & $5 \mathrm{f}$ \\
$\mathbf{2 j}$ & $2-\mathrm{HOC}_{6} \mathrm{H}_{4}$ & 80 & 71 & $5 \mathrm{f}$ \\
\hline
\end{tabular}

${ }^{\mathrm{a}}$ The yield assayed by ${ }^{31} \mathrm{P}$ NMR was always much higher, usually more than $90 \%$.

The reaction of $N$-(triphenylmethyl)alkanimines with phosphonic acid in acetic anhydride proceeds slightly more selective than when phosphorus trichloride in acetic acid was used. In the first case, we observed only a few percent of disubstituted products (iminobisphosphonic acids) ${ }^{6}$ assayed by means of ${ }^{31} \mathrm{P} \mathrm{NMR}$; whereas, in the second case, when a mixture of phosphorus trichloride and acetic acid was applied, which is obviously much "harder" phosphonylating reagent, we observed as much as $8-12 \%^{7}$ of disubstituted products. Nonetheless, after hydrolysis and typical workup procedure pure crystalline 1-aminoalkylphosphonic acids were isolated in good yields. Interestingly, when we applied the same reaction to the simplest $N$-tritylated alkanimine - $N$-(triphenylmethyl)methanimine, we found by means of ${ }^{1} \mathrm{H}$ and ${ }^{31} \mathrm{P}$ NMR that the crude reaction mixtures contain all possible aminoalkylated products, namely: aminomethylphosphonic acid (main product), iminobis(methylphosphonic) acid and nitrilotris(methylphosphonic) acid, but mainly aminomethylphosphonic acid (Scheme 2.).

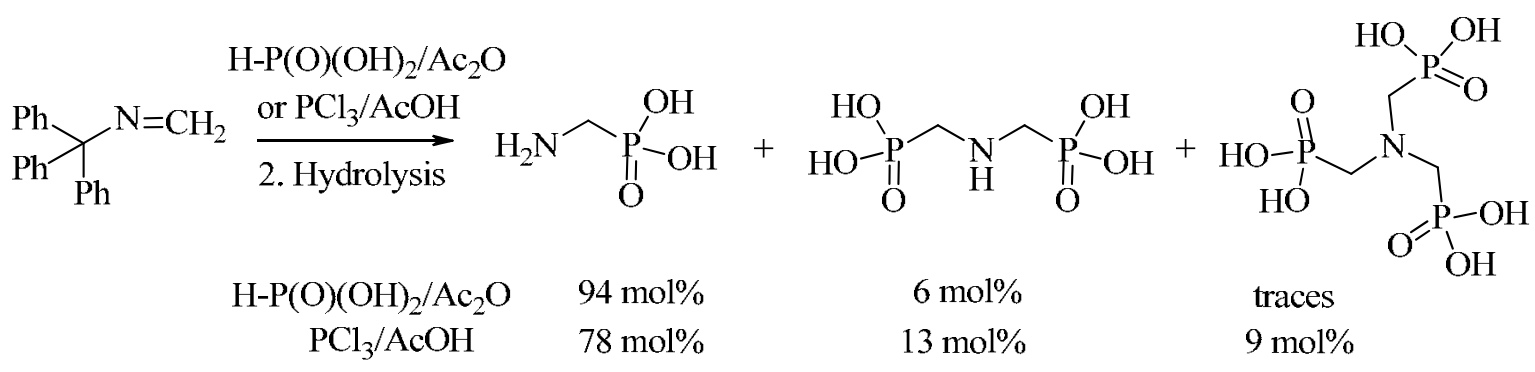

\section{Scheme 2}

It is worthy of note, that according to the literature data, aminoalkylation or amidoalkylation reactions of tervalent phosphorus compound by any kind of Mannich base derived from formaldehyde, give only perphosphonomethylated products. ${ }^{8}$ For example, an ammonia gives only the nitrilotris(methylphosphonic) acid. Once again, a reaction of $N$ (triphenylmethyl)methanimine with a mixture of phosphonic acid and acetic anhydride gave more selective results - the main product was aminomethylphosphonic acid. ${ }^{9}$ After short hydrolysis we were able to isolate pure crystalline aminomethylphosphonic acid by simple crystallization (Table).

These results clearly indicate that nucleophilic attack of phosphonylating agent (we believe that it is "acetyl phosphite"10) on acyliminium ions generated "in situ" by detritylation of $\mathrm{N}$ - 
(triphenylmethyl)alkanimine is faster than any side reactions, for example self-condensation, polymerization or decomposition of iminium salts.

Redmore $^{4 \mathrm{a}, 4 \mathrm{~b}}$ investigated the reaction of some $\mathrm{N}$-alkylalkanimines with phosphonic (phosphorous) acid, and found that reasonable results could be obtained only for imines prepared from aromatic aldehydes, which give rather stable iminium ions, because of their benzylic character. However, when he tried to use imines prepared from aliphatic aldehydes, he obtained only secondary amines - products of reduction of imines, where the phosphonic acid serves as hydride ion source.

Under such circumstances we tried to apply Redmore's protocol in the reaction of phosphonic acid with $N$-(triphenylmethyl)alkanimine, what in fact could simplify the whole procedure. Unfortunately, when we mixed phosphonic acid with the simplest $N$-trityl alkanimine, namely $N$-(triphenylmethyl)methanimine in aprotic solvents under anhydrous conditions, we neither isolate aminomethylphosphonic acid nor any product of the reduction of $N$ (triphenylmethyl)methanimine. The only product was a salt of $N$-(triphenylmethyl)methanimine with phosphonic acid, which was isolated with nearly quantitative yield. ${ }^{11}$ Our experiments also confirmed the known fact, that the so-called "phosphorous acid" exists only as a nonnucleophilic phosphonic acid, ${ }^{12}$ which contains the H-P bond. Thereafter, this reagent must be always activated to more nucleophilic form by addition of some Brönsted acid, acetic anhydride or acetyl chloride, as we have done in this work.

\section{Conclusions}

In summary, the reaction of phosphorus trichloride in acetic acid or phosphonic acid in acetic anhydride with $\mathrm{N}$-(triphenylmethyl)alkanimines is a very useful method of preparation of 1aminoalkylphosphonic acids, as well as their N-acetyl derivatives. The starting N(triphenylmethyl)alkanimines are easy available $^{1 \mathrm{a}}$ from inexpensive triphenylmethylamine (tritylamine). ${ }^{13}$ Moreover, the trityl group could be easily re-circulated. We produced large amounts of 1-aminoalkylphosphonic acids and circulated many kilograms of the trityl group without perceptible loss of it. In our hands it was the most economical method of preparation of 1-aminoalkylphosphonic acids, therefore it is in part a subject of our Polish patent. ${ }^{14}$

\section{Experimental Section}

General. NMR spectra were recorded on a Bruker Avance $300 \mathrm{MHz}$ spectrometer locked on deuterium from solvent $\left(\mathrm{D}_{2} \mathrm{O}\right.$ was used). Chemical shifts $(\delta[\mathrm{ppm}])$ were calculated from chemical shift of deuterium lock and were not calibrated. All reagents and solvents were of commercial quality. The $N$-(triphenylmethyl)alkanimines were prepared essentially as described in our first paper $^{1 \mathrm{a}}$. The triphenylmethylamine was prepared as described in the reference 13 . The 
1-aminoalkylphosphonic acids used as standards were from our collection. Most of them are also available from the Acros Catalogue [www.acros.com] (see for example: 29164, 29165, 34477, 34478, 34481, 34479, 34491).

\section{Preparation of triphenylmethylamine ${ }^{13}$}

Finely powdered solid chlorotriphenylmethane (trityl chloride) ( $28 \mathrm{~g}, 0.10$ mole) was introduced in small portions to a vigorously stirred solution of ammonium chloride (10.8 $\mathrm{g}, 0.20 \mathrm{~mol})$ in $25 \%$ of aqueous ammonia $\left(100 \mathrm{~cm}^{3}\right)$ at the temperature about $0{ }^{\circ} \mathrm{C}$ (ice-water bath). Then, the reaction mixture was stirred 12-24 hrs, white precipitate was removed by suction, washed with water $\left(5 \times 20 \mathrm{~cm}^{3}\right)$, and dried "on air" to give a crude $\mathrm{Ph}_{3} \mathrm{CNH}_{2}$ which contains about $10-15 \%$ of $\mathrm{Ph}_{3} \mathrm{COH}$. If a practically pure $\mathrm{Ph}_{3} \mathrm{CNH}_{2}$ is needed, the following procedure is recommended: $\mathrm{A}$ solution of chlorotriphenylmethane $(278.5 \mathrm{~g}, 1.00 \mathrm{~mol})$ in toluene $\left(1000 \mathrm{~cm}^{3}\right)$ was introduced dropwise to a vigorously stirred solution of ammonium chloride (saturated, but at least $53.5 \mathrm{~g}$, $1.0 \mathrm{~mol})$ in $25 \%$ of aqueous ammonia $\left(1000 \mathrm{~cm}^{3}\right)$ at temperature about $0{ }^{\circ} \mathrm{C}$ (ice-water bath). Then, the reaction mixture was stirred vigorously for about $24 \mathrm{hrs}$ at the temperature about 20 ${ }^{\circ} \mathrm{C}$, organic phase was separated, dried over $\mathrm{Na}_{2} \mathrm{SO}_{4}$, then after removing of inorganic salt by suction, a solvent was evaporated under "vacuo" from water bath at the temperature below 70 ${ }^{\circ} \mathrm{C}$. The warm oily residue was stirred in the same flask on rotavap, and hexane (about $800 \mathrm{~cm}^{3}$ ) was introduced portion-wise to cause a crystallization of $\mathrm{Ph}_{3} \mathrm{CNH}_{2}$. The crystallizing mixture was stirred additionally for about 30 minutes, then the crystalline precipitate was removed by suction, washed with a cold hexane $\left(3 \times 100 \mathrm{~cm}^{3}\right)$, dried "on air" to give 233-246 $\mathrm{g}$ (90-95\% of yield) of triphenylmethylamine with purity between $95-98 \%$ (assayed by ${ }^{1} \mathrm{H}$ NMR or by titration with $\mathrm{HClO}_{4}$ in water/acetone). Pure triphenylmethylamine could be obtained by precipitation of its hydrochloride.

\section{Synthesis of 1 aminoalkylphosphonic acids. General procedure A}

Phosphorus trichloride $\left(8.8 \mathrm{~cm}^{3}, 0.10 \mathrm{~mol}\right)$ was added drop-wise to acetic acid $\left(50 \mathrm{~cm}^{3}\right)$ at temperature below $20{ }^{\circ} \mathrm{C}$ (a cold water bath was used), then the solution was stirred additionally for about 30 minutes, cooled to about $0{ }^{\circ} \mathrm{C}$ (ice-water bath) and finely powdered $\mathrm{N}$ (triphenylmethyl)alkanimine $(0.10 \mathrm{~mol})$ was added portion-wise with vigorous stirring (PTFE stirring shaft with blade was used). The reaction mixture was stirred additionally for about 30 minutes at the same temperature, then it was gradually heated to about $100{ }^{\circ} \mathrm{C}$ (water bath) under reflux condenser connected to the $\mathrm{HCl}$ absorber. After about $1 \mathrm{hr}$ of heating, the volatile material was removed by evaporation, and oily residue was treated with water $\left(100 \mathrm{~cm}^{3}\right)$ and stirred for about 15 minutes. The precipitated triphenylmethanol was separated by suction and washed with water $\left(4 \quad \mathrm{x} \quad 10 \mathrm{~cm}^{3}\right)$. Collected filtrates were evaporated to give crude oily 1acetylaminoalkylphosphonic acids $\mathbf{1 a - j}$ (identified by means of ${ }^{1} \mathrm{H}$ and ${ }^{31} \mathrm{P}$ NMR). Oily residue was treated with $6 \mathrm{M}$ of hydrochloric acid $\left(200 \mathrm{~cm}^{3}\right)$, and refluxed gently for about $8 \mathrm{hrs}$. The hydrolysate was evaporated, residue was dissolved in methanol $\left(50 \mathrm{~cm}^{3}\right)$ and the 1aminoalkylphosphonic acid was precipitated by drop-wise addition of methyloxirane (propylene 
oxide) (about $7 \mathrm{~cm}^{3}$ ). The crystallizing mixture was kept overnight at the temperature about -20 ${ }^{\circ} \mathrm{C}$, then precipitated product was separated by suction, washed with methanol $\left(5 \times 10 \mathrm{~cm}^{3}\right)$ and dried "on air" to give the corresponding 1-aminoalkylphosphonic acid.

\section{Synthesis of 1 aminoalkylphosphonic acids. General procedure B}

Phosphonic acid $(8.2 \mathrm{~g}, 0.10 \mathrm{~mol})$ was dissolved in acetic anhydride $\left(50 \mathrm{~cm}^{3}\right)$ at the temperature about $20{ }^{\circ} \mathrm{C}$, then the solution was cooled to about $0{ }^{\circ} \mathrm{C}$ (ice-water bath) and $\mathrm{N}$ (triphenylmethyl)alkanimine was added portion-wise with vigorous stirring. After analogous procedure as it was described in the procedure $\mathrm{A}$, the corresponding 1-aminoalkylphosphonic acid was isolated.

Aminomethylphosphonic acid (2a). In the procedure $\mathrm{A}\left(\mathrm{HP}(\mathrm{O})(\mathrm{OH})_{2} / \mathrm{Ac}_{2} \mathrm{O}\right)$ the hydrolysate contained a mixture of $\mathrm{H}_{2} \mathrm{NCH}_{2} \mathrm{PO}_{3} \mathrm{H}_{2}$, and $\mathrm{HN}\left(\mathrm{CH}_{2} \mathrm{PO}_{3} \mathrm{H}_{2}\right)_{2}$ in molar ratio: 94:6 assayed by ${ }^{31} \mathrm{P}$ NMR $\left(121 \mathrm{MHz}, \mathrm{D}_{2} \mathrm{O}\right): \delta=13.3$ and 10.7 respectively. In the procedure $\mathrm{B}\left(\mathrm{PCl}_{3} / \mathrm{AcOH}\right)$ the hydrolysate contained a mixture of $\mathrm{H}_{2} \mathrm{NCH}_{2} \mathrm{PO}_{3} \mathrm{H}_{2}, \mathrm{HN}\left(\mathrm{CH}_{2} \mathrm{PO}_{3} \mathrm{H}_{2}\right)_{2}$ and $\mathrm{N}\left(\mathrm{CH}_{2} \mathrm{PO}_{3} \mathrm{H}_{2}\right)_{3}$ in molar ratio: 78:13:9 assayed by ${ }^{31} \mathrm{P}$ NMR $\left(121 \mathrm{MHz}, \mathrm{D}_{2} \mathrm{O}\right): \delta=13.3,10.7$, and 9.0 respectively; and by ${ }^{1} \mathrm{H}$ NMR $\left(300 \mathrm{MHz}, \mathrm{D}_{2} \mathrm{O}\right): \delta=2.97\left(\mathrm{~d}, J=13.1 \mathrm{~Hz}, 2 \mathrm{H}, \mathrm{CH}_{2}\right), 3.19(\mathrm{~d}, J=12.7 \mathrm{~Hz}, 4 \mathrm{H}$, $\left.\mathrm{CH}_{2}\right)$, and $3.61\left(\mathrm{~d}, J=12.9 \mathrm{~Hz}, 6 \mathrm{H}, \mathrm{CH}_{2}\right)$ respectively. After precipitation and recrystallization as it was described before, a pure aminomethylphosphonic acid was isolated.

1-Acetylaminoethylphosphonic acid (1b). ${ }^{1} \mathrm{H}$ NMR $\left(300 \mathrm{MHz}, \mathrm{D}_{2} \mathrm{O}\right): \delta=1.1$ (dd, $J=7.4 \mathrm{~Hz}, J$ $\left.=16.5 \mathrm{~Hz}, 3 \mathrm{H}, \mathrm{CH}_{3}\right), 1.8\left(\mathrm{~s}, 3 \mathrm{H}, \mathrm{CH}_{3} \mathrm{CO}\right), 4.0$ (dq, $\left.J=7.4 \mathrm{~Hz}, J=14.7 \mathrm{~Hz}, 1 \mathrm{H}, \mathrm{CH}-\mathrm{P}\right)$.

${ }^{31} \mathrm{P}$ NMR $\left(121 \mathrm{MHz}, \mathrm{D}_{2} \mathrm{O}\right): \delta=24.2$.

1-Aminoethylphosphonic acid (2b). ${ }^{1} \mathrm{H} \mathrm{NMR}\left(300 \mathrm{MHz}, \mathrm{D}_{2} \mathrm{O}\right): \delta=1.16(\mathrm{dd}, J=7.3 \mathrm{~Hz}, J=$ $\left.15.8 \mathrm{~Hz}, 3 \mathrm{H}, \mathrm{CH}_{3}\right), 3.22(\mathrm{dq}, J=7.2 \mathrm{~Hz}, J=14.3 \mathrm{~Hz}, 1 \mathrm{H}, \mathrm{CH}-\mathrm{P})$.

${ }^{31} \mathrm{P}$ NMR (121 MHz, $\left.\mathrm{D}_{2} \mathrm{O}\right): \delta=17.7$.

1-Acetylaminopropylphosphonic acid (1c). ${ }^{1} \mathrm{H} \mathrm{NMR}\left(300 \mathrm{MHz}, \mathrm{D}_{2} \mathrm{O}\right): \delta=0.70$ (t, $J=7.3 \mathrm{~Hz}$, $\left.3 \mathrm{H}, \mathrm{CH}_{3}\right), 1.25-1.44\left(\mathrm{~m}, 1 \mathrm{H}, \underline{\mathrm{CH}}_{\underline{a}} \mathrm{H}_{\mathrm{b}}\right), 1.57-1.71\left(\mathrm{~m}, 1 \mathrm{H}, \mathrm{CH}_{\mathrm{a}} \underline{\mathrm{H}}_{\mathrm{b}}\right.$ ), $1.8\left(\mathrm{~s}, 3 \mathrm{H}, \mathrm{CH}_{3} \mathrm{CO}\right), 3.80$ (ddd, $J=3.3 \mathrm{~Hz}, J=11.4 \mathrm{~Hz}, J=14,9 \mathrm{~Hz}, 1 \mathrm{H}, \mathrm{CH}-\mathrm{P})$.

${ }^{31} \mathrm{P}$ NMR $\left(121 \mathrm{MHz}, \mathrm{D}_{2} \mathrm{O}\right): \delta=23.6$.

1-Aminopropylphosphonic acid (2c). ${ }^{1} \mathrm{H}$ NMR $\left(300 \mathrm{MHz}, \mathrm{D}_{2} \mathrm{O}\right): \delta=0.95(\mathrm{t}, J=7.5 \mathrm{~Hz}, 3 \mathrm{H}$, $\mathrm{CH}_{3}$ ), 1.55-1.69 (m, 1H, $\underline{\mathrm{CH}_{\underline{a}}} \mathrm{H}_{\mathrm{b}}$ ), $1.72-1.90\left(\mathrm{~m}, 1 \mathrm{H}, \mathrm{CH}_{a} \underline{\mathrm{H}_{\mathrm{b}}}\right), 3.04$ (ddd, $J=5.2 \mathrm{~Hz}, J=8.8 \mathrm{~Hz}, J$ $=13.9 \mathrm{~Hz} 1 \mathrm{H}, \mathrm{CH}-\mathrm{P})$.

${ }^{31} \mathrm{P}$ NMR $\left(121 \mathrm{MHz}, \mathrm{D}_{2} \mathrm{O}\right): \delta=14.7$.

1-Acetylaminobutylphosphonic acid (1d). ${ }^{1} \mathrm{H}$ NMR $\left(300 \mathrm{MHz}, \mathrm{D}_{2} \mathrm{O}\right): \delta=0.74(\mathrm{t}, J=7.3 \mathrm{~Hz}$, $\left.3 \mathrm{H}, \mathrm{CH}_{3}\right), 1.15\left(\mathrm{~m}, 1 \mathrm{H}, \underline{\mathrm{CH}}_{a} \mathrm{H}_{b} \mathrm{CH}_{2} \mathrm{CHP}\right), 1.29\left(\mathrm{~m}, 1 \mathrm{H}, \mathrm{CH}_{a} \underline{\mathrm{H}}_{b} \mathrm{CH}_{2} \mathrm{CHP}\right), 1.46(\mathrm{~m}, 1 \mathrm{H}$, $\left.\underline{\mathrm{CH}_{\underline{a}}} \mathrm{H}_{\mathrm{b}} \mathrm{CHP}\right), 1.61\left(\mathrm{~m}, 1 \mathrm{H}, \mathrm{CH}_{\mathrm{a}} \underline{\mathrm{H}_{b}} \mathrm{CHP}\right), 1.89$ (s, 3H, $\mathrm{CH}_{3} \mathrm{CO}$ ), 3.96 (ddd, $J=2.5 \mathrm{~Hz}, J=12.0 \mathrm{~Hz}$, $J=14.7 \mathrm{~Hz}, 1 \mathrm{H}, \mathrm{CH}-\mathrm{P})$.

${ }^{31} \mathrm{P}$ NMR (121 MHz, $\left.\mathrm{D}_{2} \mathrm{O}\right): \delta=23.1$. 
1-Aminobutylphosphonic acid (2d). ${ }^{1} \mathrm{H} \mathrm{NMR}\left(300 \mathrm{MHz}, \mathrm{D}_{2} \mathrm{O}+\mathrm{D}_{2} \mathrm{SO}_{4}\right): \delta=0.49(\mathrm{t}, J=7.3 \mathrm{~Hz}$, $3 \mathrm{H}, \mathrm{CH}_{3}$ ), 1.03 (m, 2H, $\underline{\mathrm{CH}_{2}} \mathrm{CH}_{2} \mathrm{CHP}$ ), 1.4-1.5 (m, 2H, $\left.\underline{\mathrm{CH}_{2}} \mathrm{CHP}\right), 3.00$ (ddd, $J=5.7 \mathrm{~Hz}, J=8.6$ $\mathrm{Hz}, J=13.7 \mathrm{~Hz}, 1 \mathrm{H}, \mathrm{CH}-\mathrm{P})$.

${ }^{31} \mathrm{P}$ NMR (121 MHz, $\left.\mathrm{D}_{2} \mathrm{O}+\mathrm{D}_{2} \mathrm{SO}_{4}\right): \delta=17.6$.

1-Acetylamino-2-methylpropylphosphonic acid (1e). ${ }^{1} \mathrm{H}$ NMR $\left(300 \mathrm{MHz}, \mathrm{D}_{2} \mathrm{O}\right): \delta=0.79(\mathrm{~d}, J$ $\left.=6.6 \mathrm{~Hz}, 3 \mathrm{H}, \mathrm{CH}_{3}\right), 0.87\left(\mathrm{~d}, J=5.9 \mathrm{~Hz}, 3 \mathrm{H}, \mathrm{CH}_{3}\right), 1.88\left(\mathrm{~s}, 3 \mathrm{H}, \mathrm{CH}_{3} \mathrm{CO}\right), 1.91-2.03(\mathrm{~m}, 1 \mathrm{H}, \mathrm{CH})$, $3.87(\mathrm{dd}, J=5.5 \mathrm{~Hz}, J=17.5 \mathrm{~Hz}, 1 \mathrm{H}, \mathrm{CH}-\mathrm{P})$.

${ }^{31} \mathrm{P}$ NMR $\left(121 \mathrm{MHz}, \mathrm{D}_{2} \mathrm{O}\right): \delta=23.1$.

1-Amino-2-methylpropylphosphonic acid (2e) . ${ }^{1} \mathrm{H}$ NMR $\left(300 \mathrm{MHz}, \mathrm{D}_{2} \mathrm{O}\right): \delta=0.95(\mathrm{~d}, J=6.9$, $\left.3 \mathrm{H}, \mathrm{CH}_{3}\right), 0.99\left(\mathrm{~d}, J=6.9,3 \mathrm{H}, \mathrm{CH}_{3}\right), 2.11(\mathrm{~m}, 1 \mathrm{H}, \mathrm{CH}), 2.96(\mathrm{dd}, J=6.3 \mathrm{~Hz}, J=14.1 \mathrm{~Hz}, 1 \mathrm{H}$, CH-P).

${ }^{31} \mathrm{P}$ NMR (121 MHz, $\left.\mathrm{D}_{2} \mathrm{O}\right): \delta=14.0$.

1-Acetylaminopentylphosphonic acid (1f). ${ }^{1} \mathrm{H} \mathrm{NMR}\left(300 \mathrm{MHz}, \mathrm{D}_{2} \mathrm{O}\right): \delta=0.69(\mathrm{t}, J=6.4 \mathrm{~Hz}$, $\left.3 \mathrm{H}, \mathrm{CH}_{3}\right), 1.0-1.3\left(\mathrm{~m}, 4 \mathrm{H}, \underline{\mathrm{CH}}_{2} \underline{\mathrm{CH}}_{2} \mathrm{CH}_{2} \mathrm{CHP}\right), 1.42\left(\mathrm{~m}, 1 \mathrm{H}, \underline{\mathrm{CH}}_{\mathrm{a}} \mathrm{H}_{\mathrm{b}} \mathrm{CHP}\right), 1.65$ (m, 1H, $\mathrm{CH}_{\mathrm{a}} \underline{\mathrm{H}}_{\underline{b}} \mathrm{CHP}$ ), 1.86 (s, 3H, $\mathrm{CH}_{3} \mathrm{CO}$ ), 3.91 (ddd, $J=2.8 \mathrm{~Hz}, J=11.8 \mathrm{~Hz}, J=14.9 \mathrm{~Hz}, 1 \mathrm{H}, \mathrm{CH}-\mathrm{P}$ ).

${ }^{31} \mathrm{P}$ NMR (121 MHz, $\left.\mathrm{D}_{2} \mathrm{O}\right): \delta=23.2$.

1-Aminopentylphosphonic acid (2f). ${ }^{1} \mathrm{H} \mathrm{NMR}\left(300 \mathrm{MHz}, \mathrm{D}_{2} \mathrm{O}\right): \delta=0.47$ (t, $J=7.2 \mathrm{~Hz}, 3 \mathrm{H}$, $\mathrm{CH}_{3}$ ), 0.7-1.2 (m, 4H, $\left.\underline{\mathrm{CH}}_{2} \underline{\mathrm{CH}_{2}} \underline{\mathrm{CH}}_{2} \mathrm{CHP}\right), 1.4-1.6$ (m, 2H, $\underline{\mathrm{CH}}_{2} \mathrm{CHP}$ ), 3.00 (ddd, $J=8.1 \mathrm{~Hz}, J=$ $5.8 \mathrm{~Hz}, J=14.0 \mathrm{~Hz}, 1 \mathrm{H}, \mathrm{CH}-\mathrm{P})$.

${ }^{31} \mathrm{P}$ NMR $\left(121 \mathrm{MHz}, \mathrm{D}_{2} \mathrm{O}\right): \delta=17.7$.

1-Acetylamino-2-ethylbutylphosphonic acid (1g). ${ }^{1} \mathrm{H}$ NMR $\left(300 \mathrm{MHz}, \mathrm{D}_{2} \mathrm{O}\right): \delta=0.74(\mathrm{t}$, $\left.J=7.1 \mathrm{~Hz}, 6 \mathrm{H}, \mathrm{CH}_{3}\right), 1.0-1.2\left(\mathrm{~m}, 2 \mathrm{H}, \mathrm{CH}_{2}\right), 1.37\left(\mathrm{~m}, 1 \mathrm{H}, \underline{\mathrm{CH}}_{\underline{a}} \mathrm{H}_{\mathrm{b}}\right), 1.52\left(\mathrm{~m}, 2 \mathrm{H}, \mathrm{CH}_{\mathrm{a}} \underline{\mathrm{H}}_{\underline{b}}+\mathrm{CH}\right), 1.90$ (s, 3H, $\left.\mathrm{CH}_{3} \mathrm{CO}\right), 4.15$ (dd, $\left.J=4.1 \mathrm{~Hz}, J=18.2 \mathrm{~Hz}, 1 \mathrm{H}, \mathrm{CH}-\mathrm{P}\right)$.

${ }^{31} \mathrm{P}$ NMR (121 MHz, $\left.\mathrm{D}_{2} \mathrm{O}\right): \delta=23.7$.

1-Amino-2-ethylbutylphosphonic acid (2g). ${ }^{1} \mathrm{H}$ NMR $\left(300 \mathrm{MHz}, \mathrm{D}_{2} \mathrm{O}\right): \delta=0.51(\mathrm{t}, J=7.6 \mathrm{~Hz}$, $\left.3 \mathrm{H}, \mathrm{CH}_{3}\right), 0.54\left(\mathrm{t}, J=8.0 \mathrm{~Hz}, 3 \mathrm{H}, \mathrm{CH}_{3}\right), 0.90\left(\mathrm{~m}, 1 \mathrm{H}, \underline{\mathrm{CH}}_{\underline{\mathrm{a}}} \mathrm{H}_{\mathrm{b}}\right), 1.10\left(\mathrm{~m}, 1 \mathrm{H}, \mathrm{CH}_{\mathrm{a}} \underline{\mathrm{H}}_{\mathrm{b}}\right), 1.12-1.4$ $\left(\mathrm{m}, 3 \mathrm{H}, \mathrm{CH}+\mathrm{CH}_{2}\right), 3.15(\mathrm{dd}, J=4.0 \mathrm{~Hz}, J=16.0 \mathrm{~Hz}, 1 \mathrm{H}, \mathrm{CH}-\mathrm{P})$.

${ }^{31} \mathrm{P}$ NMR (121 MHz, $\left.\mathrm{D}_{2} \mathrm{O}\right): \delta=17.6$.

1-Acetylamino-1-phenylmethylphosphonic acid (1h). ${ }^{1} \mathrm{H}$ NMR $\left(300 \mathrm{MHz}, \mathrm{D}_{2} \mathrm{O}\right): \delta=1.85$ (s, $3 \mathrm{H}, \mathrm{CH}_{3} \mathrm{CO}$ ), 5.02 (d, $\left.J=20.9 \mathrm{~Hz}, 1 \mathrm{H}, \mathrm{CH}-\mathrm{P}\right), 7.2$ (m, 5H, ArH).

${ }^{31} \mathrm{P}$ NMR (121 MHz, $\left.\mathrm{D}_{2} \mathrm{O}\right): \delta=18.7$.

1-Amino-1-phenylmethylphosphonic acid (2h). ${ }^{1} \mathrm{H}$ NMR $\left(300 \mathrm{MHz}, \mathrm{D}_{2} \mathrm{O}+\mathrm{D}_{2} \mathrm{SO}_{4}\right): \delta=4.12$ (d, $J=16.7 \mathrm{~Hz}, 1 \mathrm{H}, \mathrm{CH}-\mathrm{P}), 6.98$ (m, 5H, ArH).

${ }^{31} \mathrm{P}$ NMR (121 MHz, $\left.\mathrm{D}_{2} \mathrm{O}+\mathrm{D}_{2} \mathrm{SO}_{4}\right): \delta=13.5$.

1-Acetylamino-1-(4-methoxyphenyl)methylphosphonic acid (1i). ${ }^{1} \mathrm{H}$ NMR $\left(300 \mathrm{MHz}, \mathrm{D}_{2} \mathrm{O}\right)$ : $\delta=1.76\left(\mathrm{~s}, 3 \mathrm{H}, \mathrm{CH}_{3} \mathrm{CO}\right), 3.57\left(\mathrm{~s}, 3 \mathrm{H}, \mathrm{CH}_{3} \mathrm{O}\right), 5.01(\mathrm{~d}, J=20.5 \mathrm{~Hz}, 1 \mathrm{H}, \mathrm{CH}-\mathrm{P}), 6.73(\mathrm{~d}, J=8.5$ $\mathrm{Hz}, 2 \mathrm{H}, \mathrm{o}-\mathrm{ArH}), 7.14$ (d, $J=8.5 \mathrm{~Hz}, 2 \mathrm{H}, \mathrm{m}-\mathrm{ArH})$. 
${ }^{31} \mathrm{P}$ NMR (121 MHz, $\left.\mathrm{D}_{2} \mathrm{O}\right): \delta=19.0$.

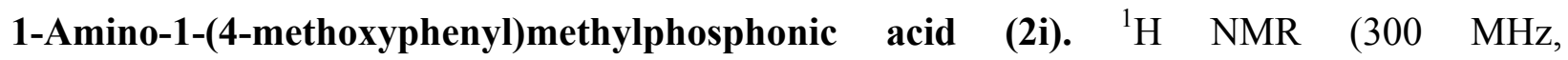
$\left.\mathrm{D}_{2} \mathrm{O}+\mathrm{D}_{2} \mathrm{SO}_{4}\right): \delta=3.31\left(\mathrm{~s}, 3 \mathrm{H}, \mathrm{CH}_{3}\right), 4.00(\mathrm{~d}, J=16.7 \mathrm{~Hz}, 1 \mathrm{H}, \mathrm{CH}-\mathrm{P}), 6.51(\mathrm{~d}, J=8.4 \mathrm{~Hz}, 2 \mathrm{H}$, o$\operatorname{ArH},), 6.9(\mathrm{~d}, J=8.4 \mathrm{~Hz}, 2 \mathrm{H}, \mathrm{m}-\mathrm{ArH})$.

${ }^{31} \mathrm{P}$ NMR $\left(121 \mathrm{MHz}, \mathrm{D}_{2} \mathrm{O}+\mathrm{D}_{2} \mathrm{SO}_{4}\right): \delta=14.0$.

1-Acetylamino-1-(2-acetoxyphenyl)methylphosphonic acid (1j). ${ }^{1} \mathrm{H}$ NMR $\left(300 \mathrm{MHz}, \mathrm{D}_{2} \mathrm{O}\right): \delta$ $=1.94\left(\mathrm{~s}, 3 \mathrm{H}, \mathrm{CH}_{3} \mathrm{CON}\right), 1.97\left(\mathrm{~s}, 3 \mathrm{H}, \mathrm{CH}_{3} \mathrm{COO}\right), 5.5(\mathrm{~d}, J=15.8 \mathrm{~Hz}, 1 \mathrm{H}, \mathrm{CH}-\mathrm{P}), 6.82(\mathrm{~d}, J=7.6$ Hz, 1H, H-6), 6.86 (t, $J=7.6 \mathrm{~Hz}, 1 \mathrm{H}, \mathrm{H}-5), 7.13$ (t, $J=7.6 \mathrm{~Hz}, 1 \mathrm{H}, \mathrm{H}-4), 7.26$ (d, J = 7.6 Hz, $1 \mathrm{H}, \mathrm{H}-3)$.

${ }^{31} \mathrm{P}$ NMR $\left(121 \mathrm{MHz}, \mathrm{D}_{2} \mathrm{O}\right): \delta=19.9$.

1-Amino-1-(2-hydroxyphenyl)methylphosphonic acid (2j). ${ }^{1} \mathrm{H} \quad \mathrm{NMR} \quad(300 \quad \mathrm{MHz}$, $\left.\mathrm{D}_{2} \mathrm{O}+\mathrm{D}_{2} \mathrm{SO}_{4}\right): \delta=4.4(\mathrm{~d}, J=17.6 \mathrm{~Hz}, 1 \mathrm{H}, \mathrm{CH}-\mathrm{P}), 6.48(\mathrm{~d}, J=7.7 \mathrm{~Hz}, 1 \mathrm{H}, \mathrm{H}-6), 6.52(\mathrm{t}, J=7.7$ $\mathrm{Hz} 1 \mathrm{H}, \mathrm{H}-5), 6.83$ (t, $J=7.7 \mathrm{~Hz}, 1 \mathrm{H}, \mathrm{H}-4), 6.89$ (d, $J=7.7 \mathrm{~Hz}, 1 \mathrm{H}, \mathrm{H}-3)$.

${ }^{31} \mathrm{P}$ NMR $\left(121 \mathrm{MHz}, \mathrm{D}_{2} \mathrm{O}+\mathrm{D}_{2} \mathrm{SO}_{4}\right): \delta=14.4$.

\section{Acknowledgements}

Financial support of our research by Polish Taxpayers is appreciated.

\section{References and Notes}

1. (a) Soroka, M.; Zygmunt, J. Synthesis 1988, 370. Later we described an application of tritylamine for an oxirane ring opening reaction. (b) Goldeman, W.; Soroka, M. Arkivoc 2003, (xii), 31.

2. For other applications of tritylamine as an ammonia equivalent see for example: (a) Sharma, S. K.; Songster, M. F.; Colpitts, T. L.; Hegyes, P.; Barany, G.; Castellino, F. J. J. Org. Chem. 1993, 58, 4993. (b) Desai, R. C. J. Org. Chem. 2001, 66, 4939. (c) Huszthy, P.; Bradshaw, J. S.; Krakowiak, K. E.; Wang, T.; Halley, N. K. Heterocycl. Chem. 1993, 5, 1197. (d) Wróblewski, A. E.; Halajewska-Wosik, A. Tetrahedron: Asymmetry 2003, 14, 3359. (e) Lake, F.; Boberg, C. Eur. J. Org. Chem. 2002, 3179. (f) Zhang, W. J.; Berglund, A.; Kao, J. L.-F.; Couty, J.-P.; Gershengorn, M. C.; Marshall, G. R. J. Am. Chem. Soc. 2003, 125, 1221. (g) Artuso, F.; Sindona, G.; Athanassopoulos, C.; Stavropoulos, G.; Papaioannou, D. Tetrahedron Lett. 1995, 36, 9309. (h) Theodorou, V.; Ragoussis, V.; Strongilos, A.; Zelepos, E.; Eleftheriou, A.; Dimitriou, M. Tetrahedron Lett. 2005, 1357. (i) Assche, I. van; Soroka, M.; Haemers, A.; Hooper, M.; Blanot, D.; Heijenoort, J. van Eur. J. Med. Chem. Chim. Ther. 1991, 26, 505. (j) Boduszek, B.; Soroka, M. Pol. J. Chem. 2002, 76, 1105. (k) Borloo, M.; Jiao, X.-Y.; Wójtowicz, H.; Rajan, P.; Verbruggen, C.; Augustyns, K.; Haemers, A. Synthesis 
1995, 1074. (1) Chen, S.; Coward, J. K. J. Org. Chem. 1998, 63, 502. (m) Sasai, H.; Arai, S.; Tahara, Y.; Shibasaki, M. J. Org. Chem. 1995, 60, 6656.

3. Other applications of phosphorus trichloride in the preparation of aminophosphonic acids were described by: (a) Engelmann, M.; Pikl, J. Pat US2304156, 1942. (b) Pikl, J. Pat US2328358, 1943. (c) Soroka, M. Liebigs Ann. Chem. 1990, 331. (d) Rendell, D. R.; Hargreaves, J. R.; Holt, B. Pat GE2135686, 1971; Chem. Abstr. 1972, 77, 140283. (e) Oleksyszyn, J.; Tyka, R.; Mastalerz, P. Synthesis 1978, 479.

4. Other applications of phosphonic acid (phosphorous acid) in the preparation of aminophosphonic acids were described by: (a) Redmore, D. J. Org. Chem. 1978, 43, 992. (b) Redmore, D. J. Org. Chem. 1978, 43, 996. (c) Oleksyszyn, J.; Gruszecka, E.; Tetrahedron Lett. 1981, 3537. (d) Soroka, M.; Jaworska, D.; Szczęsny, Z. Liebigs Ann. Chem. 1990, 1153.

5. (a) Kabachnik, M. I.; Medved', T. Y. Izv. Akad. Nauk SSSR, Ser. Khim. 1950, 635; Chem. Abstr. 1951, 45, 49728. (b) Chambers, J. R.; Isbell, A. F. J. Org. Chem. 1964, 29, 832. (c) Tyka, R. Tetrahedron Lett. 1970, 677. (d) Berry, J. P.; Isbell, A. F.; Hunt, G. E. J. Org. Chem. 1972, 37, 4396. (e) Drag, M.; Grembecka, J.; Pawełczak, M.; Kafarski, P. Eur. J. Med. Chem. 2005, 764. (f) Chalmers, M. E.; Kosolapoff, G. M. J. Am. Chem. Soc. 1953, 75, 5278.

6. Iminobisphosphonic acids were observed only for $\mathrm{R}=\mathrm{H}$ (see ref. 9), and $\mathrm{R}=\mathrm{Me}$ (4-13\% in few experiments). Other $\mathrm{N}$-(triphenylmethyl)alkanimines gave only 1-aminoalkylphosphonic acids (more than 90\% yields, assayed by ${ }^{31} \mathrm{P} \mathrm{NMR}$ ), phosphonic acid and traces of phosphorus acid.

7. About $8 \%$ for $\mathrm{R}=\mathrm{Me}$, Et and $\mathrm{Ph}$, and about $12 \%$ for $\mathrm{R}=4-\mathrm{MeOC}_{6} \mathrm{H}_{4}$. In the case of $\mathrm{R}=\mathrm{Ph}$ the structure of side product (iminobisphosphonic acid)was confirmed additionally by comparison with authentic sample prepared as in: Soroka, M.; Kołodziejczyk, K. Pat. PL187457, 2004; Chem. Abstr. 2006, 144, 51712.

8. Moedritzer, K.; Irani, R. R. J. Org. Chem. 1966, 31, 1603.

9. Crude hydrolysate contains $\mathrm{H}_{2} \mathrm{NCH}_{2} \mathrm{PO}_{3} \mathrm{H}_{2}, \mathrm{NH}\left(\mathrm{CH}_{2} \mathrm{PO}_{3} \mathrm{H}_{2}\right)_{2}$ and $\mathrm{N}\left(\mathrm{CH}_{2} \mathrm{PO}_{3} \mathrm{H}_{2}\right)_{3}$ in a molar ratio: 78:13:9, in the case of $\mathrm{PCl}_{3} / \mathrm{AcOH}$, and respectively 94:6:traces, in the case of $\mathrm{HP}(\mathrm{O})(\mathrm{OH})_{2} / \mathrm{Ac}_{2} \mathrm{O}$.

10. Acyl phosphites are described in the literature. See for example: (a) Cade, J. A.; Gerrard, W. J. Chem. Soc. 1954, 2030. (b) Nerdell, F.; Burghardt, W. Naturwissenschaften 1960, 47, 178. (c) Petrov, K. A.; Nifant'ev, E. E.; Sopikova, I. I. Dokl. Akad. Nauk SSSR 1963, 151, 859; Chem. Abstr. 1963, 59, 68654. (d) Munoz, A.; Boisdon, M.-T.; Beazier, J.-F.; Wolf, R. Bull. Soc. Chim. Fr. 1971, 4, 1424. (e) Stawinski, J.; Thelin, M. J. Chem. Soc. Perkin Trans.2 $1990,849$.

11. This salt was characterized by FTIR and NMR. IR (1/200 in KBr): 3500-2400 with max. $3464(\mathrm{NH}, \mathrm{OH}), 2367(\mathrm{H}-\mathrm{P}), 1629(\mathrm{C}=\mathrm{N}), 1251(\mathrm{P}=\mathrm{O}), 1153,1035(\mathrm{P}-\mathrm{O}) \mathrm{cm}^{-1} .{ }^{31} \mathrm{P} \mathrm{NMR}$ $\left(121 \mathrm{MHz}, \mathrm{CH}_{3} \mathrm{OH}\right.$, external lock DMSO-d $6: \delta=2.6(\mathrm{~d}, J=615 \mathrm{~Hz}) ;{ }^{31} \mathrm{P} \mathrm{NMR}\left\{{ }^{1} \mathrm{H}\right\}: \delta=2.6$.

12. Phosphonic acid (sometimes called "phosphorous acid") has tetrahedral structure with one hydrogen atom directly bound to the phosphorus, as was demonstrated by means of IR and 
Raman spectroscopy: (a) Martin, R. B. J. Am. Chem. Soc. 1959, 81, 1574; and later by ${ }^{1} \mathrm{H}$ and ${ }^{31}$ P NMR, see for example: (b) Moedritzer, K. Inorg. Chem. 1967, 6, 936. (c) Sheldrick, G. M. J. Chem. Soc. Faraday Trans. 1967, 1077. (d) Haas, T. E.; Gillman, H. D. Inorg. Chem. 1968, 7, 2051.

13. Soroka, M.; Iwańczyk, D. Pat PL195536, 2007; Chem. Abstr. 2008, 149, 176009.

14. Soroka, M.; Goldeman, W. Pat PL-195964, 2007; Chem. Abstr. 2008, 149, 513950. 